\title{
Essais
}

ESSAIS

Revue interdisciplinaire d'Humanités

$7 \mid 2015$

Normes communiquées, normes communicantes

\section{Un exemple de glissement du lexique médiatique : le sujet trans dans les productions audiovisuelles}

\section{Karine Espineira}

\section{(2) OpenEdition}

1 Journals

Édition électronique

URL : https://journals.openedition.org/essais/6238

DOI : $10.4000 /$ essais. 6238

ISSN : 2276-0970

Éditeur

École doctorale Montaigne Humanités

\section{Édition imprimée}

Date de publication : 1 décembre 2015

Pagination : 48-64

ISBN : 978-2-9544269-6-9

ISSN : 2417-4211

\section{Référence électronique}

Karine Espineira, "Un exemple de glissement du lexique médiatique : le sujet trans dans les productions audiovisuelles », Essais [En ligne], 7 | 2015, mis en ligne le 26 février 2021, consulté le 21 septembre 2021. URL : http://journals.openedition.org/essais/6238; DOI : https://doi.org/10.4000/ essais.6238 


\section{Un exemple de glissement du lexique médiatique : le sujet trans dans les productions audiovisuelles}

\section{Karine Espineira}

Cette recherche, inscrite au sein des Sciences de l'Information et de la Communication, porte son attention sur les formes de la construction médiatique des transidentités - à la lumière des études de Genre ${ }^{1}$ et des études culturelles. Elle montre que nos représentations génèrent des modélisations à la fois sociales et médiaculturelles. La notion d'Éric Maigret et d'Éric Macé proposée en 2005 dans l'ouvrage Penser les médiacultures est importante. Au néologisme de médiaculture, Macé apporte une définition dans Les imaginaires médiatiques (2006) : " ensemble des rapports sociaux et des expériences médiatisées par les représentations médiatiques et leurs usages $~^{2}$. Considérant culture des médias et culture produite par les médias, la notion abolit l'écart entre une culture "noble " et une culture dite "populaire». Sociologie de la culture et sociologie des médias sont engagées et conjuguées.

Cinq années d'observation participante du terrain transidentitaire français forment un premier socle. Le second est formé par un corpus de plus de huit cents documents audiovisuels constitué sur les bases archives l'Institut National de l'Audiovisuel (Imago, DL TV, DL Câble et Satellite, et DL Région). Ce travail se donne pour objectif de comprendre les processus de l'évolution des représentations au regard des mutations du terrain depuis les origines de la médiatisation des trans à la télévision. Le dispositif du genre y apparait le moteur du fonctionnement hégémonique d'un modèle valorisé dans le média audiovisuel et paradoxalement minoritaire sur le terrain.

1 Selon la graphie proposée par Marie-Joseph Bertini pour le terme Genre, on fera parfois usage de "la majuscule faisant signe vers les Gender Studies anglo-saxonnes " avec la volonté de contribuer à l'enrichissement de cette question au sein des SIC. "Le Gender turn, ardente obligation des sciences de l'information et de la communication françaises ", Questions de communication, $\mathrm{n}^{\circ} 15,2009$, p. 157.

2 Éric Macé, Les imaginaires médiatiques, Paris, Éditions Amsterdam, 2006, p. 12. Lire aussi : Éric Macé et Éric Maigret (éd.), Penser les médiacultures. Nouvelles pratiques et nouvelles approches de la représentation du monde, Paris, Armand Colin, 2005. 
Cette problématique trouve un début de réponse avec la mise en lumière de glissements successifs du lexique médiatique du fait-divers au fait de société pour aboutir à la notion d'égalité des droits. Les catégories proposées permettent de dépasser la classification des matériaux audiovisuels par genre au sens strict du terme. Par exemple, le traitement de type fait divers se retrouve dans des reportages de plateaux-débats et de journaux télévisés mais aussi dans les fictions policières. Il ne s'agit pas d'ignorer les spécificités et les grammaticalités de chaque genre audiovisuel mais d'observer les transversalités des thématiques et les déclinaisons de nos catégories au sein des différents genres. Les catégories " faits divers », « faits de société » et " égalité des droits » voyagent et dépassent les frontières. Dans un premier temps, ces glissements seront décrits, contextualisés et illustrés depuis les années soixante-dix à nos jours. Dans un second temps, nous montrerons qu'à chacun d'eux correspond une temporalité bien précise ; par ailleurs que la parole de l'expert en télévision doublée par des dispositifs médiatiques et sociopolitiques semble partie prenante de ce processus complexe. Le troisième temps donnera les grandes tendances de cette étude, menée entre 2008 et 2012, qui met l'accent sur les liens qu'entretiennent les représentations sociales et les représentations médiatiques devenues inséparables dans la construction médiatique du sujet trans.

\section{Du fait divers à la question de société}

L'étude des glissements sémantiques du lexique médiatique des transidentités permet de décrire et d'analyser les liens objectifs qu'entretiennent des représentations sociales et des représentations médiatiques devenues désormais consubstantielles. L'image mentale de deux arbres totalement enchevêtrés m'est venue à l'esprit. Ils ne sont pas un, mais ils ne sont plus des entités autonomes. "Importer " le concept de "consubstantialité " de Danièle Kergoat ${ }^{3}$, qui désigne l'" entrecroisement dynamique de l'ensemble des rapports sociaux ", nous permet de désigner les enchevêtrements dynamiques des représentations sociales et médiatiques. Avec la notion de "coextensivité » nous pourrions aussi décrire les processus de co-écritures et de reproduction mutuels.

Le qualificatif de "fait divers " est le premier lexique médiatique à considérer. Il décrit souvent des situations de " marginalité " des personnes trans comme les émissions des années 1970 en donnent illustration. En premier lieu, qu'est un fait divers ? Le Larousse le définit comme " un événement sans portée générale qui appartient à la vie quotidienne ", ainsi qu'une "rubrique de presse comportant des informations sans portée générale relative à des faits quotidiens ». Dans la littérature scientifique on peut s'appuyer entre autres sur

3 Danièle Kergoat, Se battre, disent-elles..., Paris, La Dispute, collection « Le genre du monde ", 2012. 
les travaux d'Annick Dubied. Elle écrit : "Tout le monde sait ce qu'est un fait divers. On en trouve partout dans les médias, et en bonne place : à la télévision, dans les journaux, à la radio, sur le Net... »" Tout en soulignant la difficulté à donner une définition, y compris dans les milieux scientifiques, elle propose une hypothèse : " le fait divers est avant tout une mise une scène sémiotique ". On ne rencontrerait pas un fait divers ailleurs que dans l'espace médiatique puisque c'est dans le processus de mise en scène qu'il semblerait se constituer. Au fait divers nous associons la notion de marginalité comme position sociale: " par rapport à une norme sociale »; le marginal est " quelqu'un qui vit en marge de la société organisée, faute de pouvoir s'y intégrer ou par refus de s'intégrer à ses normes " selon la définition des dictionnaires. Le " fait trans " (au sens de : " des personnes changent de sexe ») semble avoir pris place dans "le domaine des faits divers " dès sa première médiatisation " massive " avec la descente d'avion de Christine Jorgensen à New York en 1953 par la presse filmée 5 . Un domaine que Maurice Garçon pense immense : "Les crimes et les actes de bienfaisance, les naufrages et les sauvetages, les inondations et les épidémies, les singularités de la nature tant en rareté qu'en monstruosités, les exploits sanglants des gangsters et les mariages des têtes couronnées ou les divorces des vedettes sont matières à fait divers $»^{6}$. Le traitement médiatique des personnes trans prend place dans ce domaine du fait divers et nous proposons de résumer cette inscription avec les formules suivantes : "ici et là des individus changent de sexe " ou " des travestis à qui il arrive malheur ", dans les journaux télévisés, les séries policières et les plateaux débats.

Le fait transsexuel est notable et visible depuis la médiatisation de Christine Jorgensen ${ }^{7}$, mais reste encore exceptionnel. Il demeure sans portée générale par conséquent malgré un document inclassable du corpus datant de 1957 ("Christine Légionnaire, ou un cas difficile ») qui semble dire " le contraire » et que l'on pourrait se risquer à qualifier de « curieux ». Si l'opération de Christine Jorgensen a fait la une de la presse américaine ${ }^{8}$, popularisant cette histoire dans le monde entier, le commentaire off de ce document de l'ORTF surprend : "Ce même souci nous amène à parler des changements

4 Annick Dubied, Les dits et les scènes du fait divers, Genève/Paris, Droz, 2004, p. 13.

5 Christine Comes Home, Universal - International News, Fred Maness, États-Unis, février 1953.

6 "Introduction " à Romi, Histoire des faits divers, Pont Royal, Éditions del Duca et Laffont, 1962 ; cité par Annick Dubied dans l'article "Invasion péritextuelle et contaminations médiatiques ", Semen, n 13, Jean-Michel Adam, Thierry Herman et Gilles Lugrin (éd.), «Genres de la presse écrite et analyse de discours », 2001, p. 49.

7 Elle a été considérée comme la première personne à avoir bénéficié d'une opération de réassignation sexuelle (de « changement de sexe » est-il dit plus communément), au Danemark. Ce statut de " première transsexuelle opérée de monde » est à relativiser au regard de l'histoire de Lilli Elbe (1930) par exemple.

8 Le $1^{\text {er }}$ décembre 1952, le New York Daily News titre à sa une : «Un ex-GI devient une ravissante blonde ", le retentissement fut mondial. 
de sexe fréquents à notre époque, et particulièrement de la métamorphose de Sébastien Companys, un ancien de la Légion étrangère. Aujourd'hui ouvrier spécialisé dans une usine de Nîmes, habillé en homme il gagne sa vie en faisant un métier d'homme ; mais pendant ses loisirs, habillé en femme, il peut vivre librement. Il a bien voulu nous expliquer les circonstances de sa curieuse métamorphose ". Le mot "fréquent " qui inscrit l'acte dans une certaine quotidienneté est excessif à cette période. En effet, le $\mathrm{D}^{\mathrm{r}}$ Burou à Casablanca entame à ce moment-là une série d'opérations de réassignement de celles à qui sera conféré le titre de pionnières (Coccinelle en 1958; April Ashley et Bambi en 1960) mais elles restent exceptionnelles. Un repère fort peut être donné avec l'exemple du premier protocole de prise en charge en France datant de 1979 et ne concernait qu'une dizaine de personnes par an.

Le fait transsexuel comme fait divers, fait singulier, fait extraordinaire " aux frontières du réel » (une marginalité géographique autant qu’imaginaire) devient une "transsexualité citadine et occidentale». Les images de la descente d'avion Coccinellè à Milan en 1959 rappellent celles de Jorgensen à New York en 1953. La caméra s'attarde pareillement sur les deux femmes accueillies en célébrités. Si les pionnières semblent alors adulées, "le changement de sexe " va bientôt cesser d'être une attraction. Ce qui était confiné aux cabarets, en faisant irruption dans l'espace public et médiatique, commence à inquiéter. Le " fait transsexuel » en sortant de sa " confidentialité " devient une marginalité à la fin de l'âge d'or du cabaret transgenre dans les années 1970 ; années marquées par deux chocs pétroliers successifs, la fin de la libération sexuelle et l'impact de la crise dans les milieux populaires.

À la suite de la presse populaire, la télévision va s’intéresser à la prostitution des travestis et des transsexuelles sous l'intitulé " prostitution masculine ". Prostitués $^{10}$ est l'émission qui illustre le mieux cette tendance. Il s'agit de l'un des programmes abordant le sujet en le plaçant sous la thématique de la prostitution masculine, bien que l'on retrouve ici le descripteur transsexualité dont l'usage est encore rare dans les années 1970 . Il n'est pas le seul à traiter le sujet par ce détour. On note les précautions prises par les journalistes dont Jean-Marie Cavada : "aborder ce sujet avec tolérance ", "mettre de côté nos préjugés ». Le journaliste illustre à sa manière la logique ou l'hypothèse du "dire c'est faire " ${ }^{11}$ ou plutôt ici, " montrer c'est faire exister " : "Vous allez dire que la télévision risque de jouer une nouvelle fois un rôle d'entraînement. J'ai entre les mains une preuve contraire. Quatre jeunes ont arrêté la prostitution! ». La télévision semble ici en mesure de changer le cours des

9 Arrivée de "Coccinelle » à l'aéroport de Milan, Actualités filmées, ORTF, janvier 1959.

10 Prostitués, collection : C'est-à-dire, Antenne 2, 25 février 1976.

11 John Langshaw Austin, Quand dire, c'est faire, [1962] 1970, réédition Seuil, coll. « Points essais », 1991. 
choses, dit Cavada. Toutefois, la prostitution comme marginalité se conjugue souvent avec le fait divers dans cette rue Curiol du Marseille des années 1970 connue pour être un lieu de prostitution.

L'association entre personnes trans et homosexuelles est fréquente à la télévision. Les journaux télévisés n'y échappent pas. Le ton " fait divers " est le traitement médiatique par défaut. Il semble se caractériser par des qualificatifs tels que "dramatique », " curiosité » ou "insolite » quand le sujet n'est pas tout simplement présenté dans la rubrique "fait divers " en fin de journal télévisé. L'une des principales difficultés de travailler sur la notion de fait divers, c'est qu'elle ne se traduit pas seulement pas un genre mais aussi pas un "état d'esprit " que l'on pourrait s'évertuer à chercher ou à noter dans le ton grave d'un Jean-Louis Burgat parlant d'un « congrès d'homosexuels » (1976) ou de celui plus humoristique (voire empreint de dérision ${ }^{12}$ ) d'un Bruno Masure sur le "démantèlement d'un réseau de proxénète au bois de Boulogne " (1990), le lieu est qualifié de " camping d'un genre particulier ». Ces usages décrivent des marginalités comme la tenue d'un congrès d'homosexuels à Rome en 1976 dans le sujet "La via del missione " ${ }^{13}$. Le commentaire suivant apporte des éléments de contexte : "Étrange atmosphère que ce congrès, aussi étrange pour le moins que ces jeunes hommes habillés en femmes et jeunes femmes habillées en hommes. La France avait même délégué quelques représentants ou représentantes, on ne sait plus ». À l'image de Cavada, Jean-Louis Burgat est tout aussi précautionneux dans sa conclusion : "Même si on ne comprend pas tout, l'un des piliers de la liberté, c'est de savoir respecter les autres ". Visant la portée générale, le traitement de type " fait divers " glisse vers le "fait de société " en prétendant que cela concerne l'ensemble de la société et de ses institutions. On pourrait envisager une synonymie entre fait de société et question de société. Le fait qu'un marginal rejette les normes sociales et se retrouve en position de mendicité peut donner lieu à un traitement de fait divers. Mais le fait que " la pauvreté » conduise à la précarité et jette à la rue de nombreuses personnes donnerait lieu à un traitement de type fait de société questionnant la responsabilité et une éventuelle réponse collective. Si une personne trans est perçue comme marginale pour avoir " choisi » de changer de sexe ou de genre social, sa situation n'engage qu'elle. Mais si ces personnes deviennent de plus en plus visibles dans des situations de marginalité et de précarité, mettant en lumière l'absence de dispositifs sociaux pour les accueillir, la responsabilité devient collective et exige une réponse sociale.

12 L'interprétation est parfois une difficulté du chercheur qui ne peut être invisible à lui-même : il faut accepter, appréhender et étudier les effets de visionnage sachant que l'interprétation débordera toujours l'intention. Dans cet exemple, il est difficile de trancher entre humour, dérision, voire ironie sans interroger la justesse et la partialité de " ma " traduction.

13 Congrès homosexuels, collection : IT1 Nuit, TF1, 25 avril 1976. 
Le glissement du "fait trans " (le fait divers) vers « la question trans " (le fait et la question de société) sur laquelle l'ensemble des téléspectateurs est invité à prendre la mesure semble s'opérer paradoxalement avec les mêmes émissions traitant de la prostitution masculine comme illustré avec l'émission Prostitués. À l'image d'un journal télévisé composé de différentes rubriques (informations politiques, économiques, culturelles, faits divers, etc.), les dispositifs des émissions alternent débats plateaux et reportages comme autant de segments autonomes et complémentaires. Un genre télévisé peut ainsi être traversé par plusieurs de nos catégories (fait divers dans les reportages et fait de société dans le débat). Au-delà des images sombres de la prostitution filmée de nuit, les discours s'orientent vers l'impossibilité pour les personnes trans de changer leur état civil ou d'être acceptées par l'ensemble de la société. Le discours global dans les programmes des années 1970 peut se traduire ainsi : la marginalisation conduit les travestis et les transsexuels à la prostitution, à la prise de stupéfiant et les plonge dans le «monde trouble de la pègre».

Dans l'un de ces programmes, intitulé "Pour une dose d'enfer " ${ }^{14}$, on constate que si l'on s'éloigne du registre du fait divers, celui du fait de société émergent ne se défait pas totalement du thème de la marginalité. L'émission illustre à la fois un glissement sur l'idée d'une marginalité non-choisie et des assimilations à la prostitution. L'adresse convenue et précautionneuse disparaît tandis que la dénomination " prostitution masculine " perdure. Ce programme, présenté comme une enquête sur l'univers des stupéfiants associe drogue et travestis à Marseille. La ville est présentée comme la ville du grand banditisme. L'examen du « fléau social » devant interroger la société dans son ensemble ne se départit pas pour autant des lieux communs. Au contraire, de nouveaux sont construits donnant lieu à de nouvelles assimilations avec les milieux de la pègre et de la drogue. Le corpus est très riche de la parole des personnes trans dénonçant les assimilations à la marge, aux milieux troubles.

Dans les années 1980, le traitement médiatique de type "fait divers " semble diminuer au profit de celui de " fait de société » avec l'actualité (Platean René Küss, 1982), le documentaire (Le corps de mon identité, 1983), le plateau de débat (collections: Les dossiers de l'écran, 1987 ; En quête de vérité, 1992; Bas les masques, 1995, Ce qui fait débat, 2001), le talk-show (collections : Ciel mon mardi, Ça se discute, Français si vous saviez, C'est quoi l'amour ?) sur l'ensemble des deux décennies suivantes (1989-2009). Dans un premier temps l'augmentation des traitements de type fait de société s'explique par l'arrivée importante de médecins (généralistes, urologues, endocrinologues, chirurgiens et psychiatres) et de juristes (avocats, juges, procureurs) sur les plateaux de télévision. Ils viennent expliquer «le mystère " que représente la transsexualité dont ils s'accordent pour dire que la science ne parvient pas à

14 Pour une dose d'enfer, collection : Les mercredis de l'information, TF1, 15 décembre 1981. 
l'expliquer. Dans un second temps, on doit considérer les développements des émissions, des genres journalistiques, l'émergence de nouvelles chaînes qui participent et contribuent à la popularisation du sujet.

Ce glissement - on l'a dit, plus marqué - coïncide avec l'apparition d'une nouvelle classe d'experts en télévision, dont les psychiatres. Les questions sont qualifiées d'éthiques. La société interroge à travers eux les progrès techniques, leurs conséquences sociales et leur introduction dans le champ bioéthique. Les travaux de Dominique Mehl sur les débuts de la controverse bioéthique ${ }^{15}$ sont éclairants. Les mêmes analyses appliquées au transsexualisme (comme concept et pratique) et au transgenre (comme expression identitaire culturelle multiple et transversale) montrent que les représentations de la nature, de l'ordre et de l'agencement des genres masculin et féminin sont aussi bousculées par ce biais. Le corps médical qui plaide à la télévision valide l'autorité de l'expert psychiatre en l'inscrivant au sein d'un réseau auto-légitimant. Les experts de la question trans ne se contredisent jamais entre eux dans les médias et aux yeux du grand public profane.

La télévision des années 1980 ne se contente plus d'un généraliste ou d'un urologue. Les personnes trans se retrouvent sur les plateaux de télévision en présence de nombreux experts, non seulement de la médecine mais aussi de la justice, pour penser « la question transsexuelle». Le plateau de débat à plusieurs voix illustre cette évolution. Un premier grand plateau marque cette tendance : D'un sexe à l'autre, elle ou lui ? (Collection : Les dossiers de l'écran, 1987). Personnes trans et experts se font face. La question de société est la suivante : traiter les transsexuels et les aider à s'insérer. Les experts sont nombreux : «le Professeur René Küss, urologue, président de l'Académie nationale de médecine, qui a dirigé un groupe de travail sur le transsexualisme »; "le Professeur Jean-Pierre Luton, endocrinologue à L'Hôpital Cochin "; " le Professeur Jacques Breton, neuropsychiatre "; "le Professeur Pierre Banzet, chirurgien plasticien "; "Jean Mazars, juriste ". Ces personnalités se succéderont chez Dechavanne, Bercoff, Delarue ou Dumas durant la décennie suivante, ainsi que des experts-psychiatres, psychanalystes ou sexologues : Bernard Cordier, Nadine Grafeille, Mireille Bonierbale, Catherine Millot, Marion Meney, entre autres. Les experts sont invités à comprendre et à expliquer le " mystérieux » et " inexpliqué phénomène » que représente alors la transsexualité. Le corpus met en lumière dans leurs discours les hypothèses biologiques, les vulgarisations de données physiologiques sur la détermination et la formation du sexe. Les questions sociales et psychiatriques entrent en scène avec l'évocation des problèmes très spécifiques comme l'identité, l'adéquation sexe-genre, les enjeux relationnels, l'insertion sociale et professionnelle.

15 Dominique Mehl, La bonne parole. Quand les psys plaident dans les médias, Paris, La Martinière, 2003, p. 89-98. 
Le questionnement du " fait de société " trouve des illustrations dans les récits d'opérations dites "de changement de sexe " définies comme " rectifications" (termes communs aux personnes trans et aux experts), l'inventaire des enjeux relationnels et sociaux, doublé par des dispositifs impliquant le public des téléspectateurs (« les standards svp » de la collection "Les dossiers de l'écran ", les sondages en temps réel de "Ciel mon mardi ", la parole donnée aux téléspectatrices témoins " D'aujourd'hui madame » ou du public in situ des talk-shows des productions Reservoir Prod ("Ça se discute", "J'y vais j'y vais pas", etc.) entre autres. Les dispositifs consultatifs à l'adresse du grand public pris à témoin semblent contribuer au basculement du fait divers en fait de société. Le traitement de type "fait de société " marque ainsi les décennies 80 et 90 ainsi qu'une partie des années 2000 .

\section{Égalité des droits}

Le second glissement du lexique médiatique concerne la notion d'égalité des droits inscrite dans une histoire des idées et des mentalités. L'action des minorités ne peut être ignorée : mobilisation des associations dans le cadre de la pandémie du Sida dans les années 1980, puis dans le cadre des débats sur le Pacs, la PMA, l'homoparentalité et le mariage pour tous, ou en faveur des sans-papiers, des sans-logis, des années 1990 à nos jours. L'action des opposants doit aussi être prise en compte. En 2013, on a vu avec les débats autour de la loi du mariage pour tous, qu'une démarche d'égalité des droits peut s'inscrire avec force dans nos journaux télévisés quotidiens et l'ensemble des médias sur des mois. Si le discours militant a trouvé à s'exprimer, les dérapages homophobes, en acte comme en parole, ont été d'une grande violence et ont contribué à maintenir l'attention des médias sur cette période comme on peut le constater avec les études récentes de Maxime Cervulle ${ }^{16}$ et Fred Pailler ${ }^{17}$, Maxime Cervulle et Virginie Julliard ${ }^{18}$ notamment.

L'inscription de la transidentité (terme parapluie issu du terrain dans les années 2000 pour désigner les identités trans) dans le mouvement de l'égalité des droits est médiatiquement plus confidentielle, bien que très active sur le terrain associatif. Notre première référence concerne un sujet de journal télévisé de France 3 Alsace : «Au XXI siècle, les clichés et les idées reçues sont toujours

16 Maxime Cervulle, " "Les controverses autour du "mariage pour tous" dans la presse nationale quotidienne : du différentialisme ethno-sexuel comme registre d'opposition ", L'Homme et la société, no 189-190, 2014.

17 Maxime Cervulle et Fred Pailler, "\#mariagepourtous : Twitter et la politique affective des hashtags ", Revue Française des Sciences de l'Information et de la Communication, nº 4, 2014 [en ligne] : http://rfsic.revues.org/717 [consulté le 20/01/2014].

18 Maxime Cervulle et Virginie Julliard, « "Différence des sexes” et controverses médiatiques : du débat sur la parité au "mariage pour tous" (1998-2013) ", Le Temps des médias, n²1, 2014. 
là lorsqu'un homme se sent femme, ou qu'une femme se sent homme. Malgré les récentes déclarations du Ministère de la Santé, la transsexualité est toujours considérée comme une maladie mentale. Cécile Poure et Arnaud Rapp nous proposent le témoignage de cinq personnes qui ont choisi d'être eux-mêmes, quitte à défier les lois de la nature $"{ }^{19}$ (Source : France 3 Régions). Le titre comme le descripteur font référence à la transsexualité (" les transsexuels ", " transsexualité »). On sait que le collectif strasbourgeois (STS67) au centre du sujet est radicalement opposé à ce vocabulaire estimé pathologisant. STS valorise le terme transgenre dans toutes ses acceptions. Le croisement de cette donnée du terrain avec l'intitulé du reportage est donc questionnant. Le terme " transsexuel » succéderait-il au terme travesti pour désigner les trans ? Le corpus montre qu'il n'en est rien. Les deux termes perdurent simultanément.

Ledit document se place dans la rubrique "Le Mag " du 19/20 avec le décrochage pour les informations des régions. Les premiers commentaires entrent dans le vif du sujet sur le mode de la télévision transgressive : "Le portrait de cinq personnes qui ont choisi d'être elles-mêmes "; Transsexuel, mot qui recouvre bien des fantasmes, clichés campés sur leurs talons aiguilles ». Sous forme de synthèse, ce dernier commentaire : "Derrière le maquillage outrancier des folles d'un jour, une minorité invisible le reste du temps, une minorité qui a choisi de défier les lois de la nature, les lois tout court, qui a choisi d'être soi ". Suivent cinq portraits ${ }^{20}$ : Clément (titre : FtM Mâle dans sa peau), Cassandre (titre : De l'autre côté du miroir), Pascal/Claire (titre : Un homme et une femme), Cornelia (titre : Le cri du corps : folle furieuse et fière), Alexandra (titre: No man's land). Le portrait d'Alexandra est cadré par l'intervention du psychiatre Hugues Paris. L'expert fait intrusion alors que le dispositif ne nous y préparait pas. S'agit-il d'une maladresse ou d'une mise en perspective par la télévision de deux discours considérés aussi compétents et légitimes l'un que l'autre ? La télévision transgressive manque son objectif avec le discours de l'expert venant affirmer que l'on ne dispose pas de son corps, et par là même contredire ce qu'elle prétendait montrer : la possibilité d'être soi.

La conclusion du reportage mérite que l'on s'y arrête aussi : «Hors normes mais heureux ». Les associations estiment entre 40000 et 60000 le nombre de transgenres vivant en France. Une minorité qui a l'audace de poser une question loin d'être marginale : "qu'est-ce qui définit un homme, une femme? ", sur des images d'un panneau avec les silhouettes si habituelles des toilettes pour " homme/femme » et des animations interchangeant les attributs de l'un et de l'autre (habits, barbes, cheveux longs ou courts, etc.). La dernière partie de la conclusion est la suivante : "Son sexe ou le rôle qui lui a été attribué par la

19 Titre propre : Les transsexuels, collection : 19 20. Édition Alsace, France 3 Alsace, 21 juin 2009.

20 Les commentaires en voix off sont en italique ; les dialogues entre guillemets; les descriptions entre crochets. 
société ? Une norme culturelle, à laquelle nous sommes tous et toutes soumis ". Le droit à exister dans la différence (l'égalité des droits) semble être valorisé. L'expert psychiatre convoqué dit pourtant : "la liberté du sujet ça n'existe pas ». L'exemple du reportage de France 3 à l'occasion d'une Marche des Fiertés, dans le contexte de l'égalité des droits est celui d'une transgression avortée.

Une autre approche peut être proposée avec la téléréalité qui mérite d'autres qualificatifs que trash tv dans une perspective post-critique. La conversation quotidienne dont elle est l'objet offre une occasion courante de nous intéresser aux effets symboliques. Quand un médecin généraliste, un coiffeur, un étudiant, un technicien, rapportent qu'un personnage, tel Erwan $^{21}$ dans Secret Story, a grandement intéressé leurs enfants, leurs proches et eux-mêmes, comment ne pas tendre l'oreille ? De même, quand ils confient avoir été amenés à se poser des questions ou quand ils avouent un attachement au personnage comme " personne ordinaire».

Lidée d'une approche compréhensive de la télévision autorise cette question : la téléréalité, concourt-elle au mouvement de l'égalité des droits ? Yves Chevalier questionne l'intention déclarée par des professionnels des médias comme Pascale Breugnot, directrice des programmes de TF1 dans les années 1990, concernant le rôle et la place de la téléréalité sur la chaîne : "elle affichait clairement, comme une mission de service public, l'intention de la télévision de recréer la communication entre les Français et de se substituer aux mécanismes sociopolitiques défaillants $»^{22}$. Si la déclaration d'intention va dans le sens d'une légitimation d'un genre de la télévision - ici la téléréalité les frontières entre les notions de question de société et d'égalité des droits sont floues mais elles semblent exister : il ne s'agit pas seulement de débattre (poser des questions et formuler des problématiques) mais d'agir (renouer le dialogue, agir sur le réel) depuis l'espace médiatique. Est-ce que " montrer » (au-delà des questionnements sur la sincérité des intentions déclarées ou d'une vertu supposée ou souhaitée des médias) c'est aussi contribuer ou œuvrer à l'égalité de la médiatisation et donc des droits ? Il faudrait ainsi envisager d'établir un pont entre " égalité de la média[tisa]tion » et égalité des droits pour désigner l'un des dispositifs de pouvoir structurant les relations entre individus et sociétés.

L’analyse de la téléréalité des années 2000 offre des perspectives d'études. Les dispositifs sociopolitiques sont scénarisés et agrémentés de " naturalité " avec des acteurs qui n'en sont pas sur le papier. L'individu en tant que « sujet acteur d'un quotidien » est recréé depuis Big Brother et ses déclinaisons (ÉtatsUnis, en Hollande, en Belgique, en Grèce, au Portugal, etc.). Pour la France,

21 Un candidat de la première édition de Secret Story en 2007, avec le secret : « j’ai décidé de changer de sexe $"$.

22 Yves Chevalier, Do You speak television? Apprentissage médiatiques et compétences sociales, Louvain, EME, 2006, p. 60. 
on retiendra Loft Story puis Secret Story. S'intéressant à la première version de la téléréalité made in France, Dominique Mehl contextualise en rappelant que les premières initiatives de reality shows (Psy Show, 1983 ; Perdu de vue, 1991 ; Bas les masques, 1992) avaient été " accueillies avec scepticisme par le monde lettré ». Mehl semble adopter une posture compréhensive quand elle souligne l'importance de « décrypter le sens social du message incarné par la vie du Loft et déchiffrer les raisons de l'attachement du public à ces péripéties si triviales ${ }^{23}$. La référence de Mehl à Guy Sorman, qui avait remarqué « la proximité culturelle des "lofteurs" avec le monde adolescent : même vocabulaire, mêmes références à la culture télévisuelle " fait écho. Durant une partie de notre recherche aussi bien consacrée au terrain qu'aux visionnages, nous ne pouvions manquer un recueil informel de données : " écouter et annoter le quotidien ", fait des avis des enfants des proches et des moins proches, du jeune et du moins jeune public friand de téléréalité dans notre entourage. "La force des épreuves de la quotidienneté " soulignée par Lochard et Soulez (2003) se fait entendre derrière le « réalisme de Big Brother et sa proposition de dévoilement, se profile d'abord une dimension plus sociale qu'anthropologique, puisqu'il s'agit de s'interroger sur les normes d'une société donnée plutôt que de réfléchir sur la condition humaine elle-même, comme dans la perspective orwellienne ${ }^{24}$. Cette matière télévisuelle donne à appréhender l'autre qui, à défaut d'être pleinement intime, le rend familier. La téléréalité génère-t-elle de l'ontologie en mettant en scène l'altérité ? L'inclusion (ou non-exclusion) de l'altérité semblerait être la condition de l'égalité.

Cette inscription possible des trans dans le mouvement pour l'égalité est relativement inattendue ou du moins, elle peut surprendre. Ce serait oublier le précédent de Nadia Almada, gagnante de Big Brother au Royaume-Uni en 2004. Christine Burns, l'une des responsables de l'association anglaise Press for Change, avait expliqué suite à la victoire de Nadia Almada dans Big Brother, qu' elle avait obtenu $80 \%$ des voix, ce qui constituait alors le record dans l'histoire du programme avec près de 3,8 millions de votes. Propos impensables dans le contexte associatif français, Christine Burns affichait une croyance, une télévision pédagogique. Elle confia que la personne de Nadia Almada, côtoyée quotidiennement durant des mois par les téléspectateurs, avait autant contribué pour l'acceptation des personnes trans dans la société anglaise que des années d'activisme ${ }^{25}$.

23 Dominique Mehl, "Le public de Loft Story : distance et connivence ", in MédiaMorphoses, Guy Lochard (éd.), hors série "La télé-réalité, un débat mondial ", Paris, PUF/INA, 2003, p. 134.

24 Guy Lochard et Guillaume Soulez, «Une mondialisation inachevée : limites, non frontières de la télé-réalité », in MédiaMorphoses, Guy Lochard (éd.), hors série "La télé-réalité, un débat mondial », Paris, PUF/INA, 2003, p. 150-151.

25 Karine Espineira, La transidentité, de l'espace médiatique à l'espace public, Paris, L'Harmattan, 2008, p. 94-98. 
Nous retrouvons les mêmes dispositifs et en partie mêmes effets que pour Erwan Henaux en France en 2007 : adhésion du public et des candidats, inscription dans le quotidien, la transidentité passe au second plan au profit d'une "personne attachante et courageuse " auprès du jeune public, bien qu'il ne l'emporte pas. Ce dernier apprend en quelque sorte la masculinité auprès des autres concurrents et son secret éventé (" un homme dans un corps de fille", "J'ai décidé de changer de sexe "), sans détours il dit qu'il est là pour financer son opération. Durant les mois suivant son élimination du jeu, on le verra dans Toute une histoire (France 3, 11/04/2007), $50 \mathrm{mn}$ Inside (TF1, 12/01/2008, 09/02/2008), C'est quoi l'amour? (TF1, 24/12/2010), Téléréalité, leur nouvelle vie (TF6, 30/01/2013, 06/02/2013). La toile ne manque pas de ses interviews dans les sites dédiés people, téléréalité ou encore programmes pour adolescents. Il ne serait pas inintéressant d'enquêter sur la façon dont le jeune public de 2007 perçoit les personnes trans et leurs revendications à l'égalité des droits en comparaison avec un public n'ayant pas eu d'attrait pour la téléréalité.

On retrouve cette inscription dans les sujets d'actualité. Voici l'affaire opposant Clarisse à son employeur, où se manifeste le mouvement des droits. L'affaire est abordée dans le magazine de société présenté par Thierry Guerrier. L'émission est consacrée " aux homosexuels et aux transsexuels. Plusieurs spécialistes sont invités à débattre sur le sujet, à réagir aux reportages et à répondre aux questions des téléspectateurs $»^{26}$ (Source : INA). Clarisse fait l'objet d'un reportage intitulé Licencié pour avoir changé de sexe: "Cadre dans les finances, Baptiste a changé de sexe pour devenir Clarisse, suite à la révélation de ce changement, son employeur lui a adressé une lettre de licenciement ». L'affaire figure aussi au programme des journaux télévisés en deux temps, l'information sur l'affaire puis le délibéré : 1 . médiatisation : "Clarisse est un transsexuel qui conteste son licenciement qu'il juge discriminatoire car lié à son changement de sexe ${ }^{27} ; 2$. délibéré : "Autre affaire dont nous vous avons parlé sur cette antenne, celle de Clarisse, embauchée, en tant que Baptiste comme cadre dans l'entreprise montpelliéraine Kaliop, spécialisée dans le multimédia... $»^{28}$. La transsexualité de Clarisse est rappelée comme cause du licenciement, jugé abusif par le tribunal des Prud'hommes. Le procès s'inscrit à l'évidence dans le thème de l'égalité des droits bien que resurgissent parfois les accents du fait divers, comme dans le cas récent d'un instituteur devenu institutrice ("Une institutrice change de sexe », sur France 2 en 2005) ou d'un professeur qui change de sexe ("Professeur transsexuel", sur France 3 : Pays de Loire en 2010 ; document hors corpus).

26 Titre propre : Rencontre du troisième sexe, collection : C dans l'air (numéro 21), France 5, 30 juin 2008.

27 Titre propre : Procès aux prud'hommes pour discrimination contre un transsexuel, collection : 19 20. Édition Languedoc, France 3 Languedoc Roussillon, 31 mars 2008.

28 Titre propre : Délibéré affaire Clarisse, collection : 19/20. Édition Languedoc Roussillon, France 3 Languedoc Roussillon, 9 juin 2008. 
France 3 régions, Arte ou encore LCI ont ainsi accumulé les sujets en lien avec des affaires dites ou supposées sensibles : Larmée est embarrassée par un adjudant devenu femme (LCI, 2009), Un transsexuel inscrit deux fois sur les listes électorales (France 3 Lorraine, 2009), Le mariage de Stéphanie et Élise (France 3 Lorraine, 2011) ; les manifestations: Manifestations des transsexuels à Strasbourg (Arte Info, 2005) ; la journée Idaho: Journée mondiale de lutte contre l'homophobie (France 3 Alsace, 2009) ; Les reportages à l'occasion de la marche des Fiertés : Le quotidien des personnes transsexuelles (France 3 Alsace, 2009), Lyon: Gay Pride (France 3 Rhône Alpes, 2009), Invitée : Pascale Ourbih (France 3 Édition Île-deFrance, 2009) ; d'un festival : La Transsexualité (France 3 Pays de la Loire, 2009).

Visionner et décrypter n'est pas un travail suffisant. Étudier la progression des descripteurs des fiches INA permet de noter les évolutions et les associations lexicales (par exemple : travesti et homosexuel, travesti et transsexuel, transsexualité et maladie, discrimination et homophobie, féminisme, manifestation et manifestation, prévention et peur, etc.) s'avère être une démarche opportune. La parole qui revendique l'égalité des droits, représente environ $3 \%$ de la matière télévisuelle réunie pour former notre corpus mais une étude minutieuse et spécifique des fiches pourrait être envisagée. Cette parole s'exprime essentiellement avec l'actualité en lien avec La Marche des fiertés, d'un procès médiatisé comme celui de Clarisse, l'action de terrain ou d'une ou d'un " coup médiatique " et elle est relayée essentiellement par les chaînes locales et les chaînes thématiques.

\section{La construction d'un modèle dominant}

En grande partie, dans les productions figurant dans le corpus, les renvois à "l'erreur de la nature " et à la souffrance côtoient les récits plus ou moins militants. Transitions ou "transformations " sont le plus souvent qualifiées de " parcours de combattant ». Les conséquences familiales et socioprofessionnelles sont toujours abordées. L'idée que la télévision veut et peut " défaire les mentalités » et "défaire des inégalités » fait son chemin. La perspective s'inscrit depuis le traitement d'une marginalité jusqu'au fait de société, puis l'intérêt pour des individus et des mouvements engagés dans l'égalité des droits.

La télévision parle massivement de travestis et c'est à partir de la prise en charge institutionnelle en 1979 en France que les termes transsexualité puis transsexualisme s'imposent dans les documents télédiffusés comme dans les fiches de sauvegarde à l'INA. Les transsexuels " chassent " les travestis et les médias "transsexualisent" toutes les identifications de genre trans. Pour autant, les fiches de l'INA continuent à indiquer le terme de travesti comme descripteur par défaut. Pour les commentaires de télévision, la tendance globale est la suivante : les trans changeraient de "sexe " par souci identitaire et non sexuel, bien que journalistes et experts s'évertuent à ne parler que de changement de sexe et jamais de changement de genre. 
Dans notre étude, on voit que la télévision participe à la construction d'une représentation dominante ('institué transsexe, représentation valorisée) au détriment d'une représentation dominante sur le terrain transidentitaire (l'institué transgenre, représentation minorée). Nous constatons cependant l'existence qu'une représentation tardive et toujours confidentielle pour cette dernière représentation. La modélisation est plus ou moins souple, et elle entretient une forte adéquation avec l'ordre social et historique (ici celui du Genre au sens de rapports sociaux de sexe), en faisant place à une certaine perturbation (le «trouble ", la transgression).

Dès les premiers visionnages à l'Inathèque, il ne faisait aucun doute qu'un document décontextualisé ne donnerait qu'un sens trompeur à l'étude, en ignorant logiques de production, structures de représentation de la période de production, dynamiques sociales en action, et pour tout dire un esprit $d u$ temps. Les productions des années 1970 sont imprégnées symboliquement par " la libération sexuelle ", mais aussi par une télévision sous tutelle passant du noir et blanc à la couleur, parfois très audacieuse tant dans ses choix thématiques que dans ses traitements médiatiques.

L'ensemble de l'analyse montre que la télévision formule une " transsexualisation " globale, elle est une forme d'institutionnalisation de la représentation et de la relation. Il va falloir attendre la deuxième moitié des années 2000 pour voir des documentaires s'intéressant plus à la condition des personnes trans (au collectif) qu'au récit d'un trajet individuel.

Ce que ces documents ont aussi en commun, c'est de mettre en scène des identités "paniquantes", celles qui tiennent des propos qui créent du trouble. C'est ici que "l'institué transgenre " trouve d'ailleurs à s'exprimer hors de la norme. À l'opposé, «l'institué transsexe » se plie toujours à cet ordonnancement qui « rassure ». On ne peut plus ignorer que les identités autres, alternatives, transgenres, transvariants, genderqueers (termes du terrain militant) ont aussi des effets identitaires sur les journalistes, les réalisateurs, les documentaristes mais aussi sur l'ensemble de la société. Nous proposons ici la notion de gender panic (panique de genre) pour désigner ce que révèle le traitement médiatique des sujets trans. Ce néologisme pointe vers la notion anglo-saxonne de sexpanic développée par Carole Vance, Gayle Rubin, Estelle Freedman, Jeffrey Weeks ou encore Lisa Duggan ${ }^{29}$. L'historien Allan Bérubé l'a ainsi défini comme « $a$ moral crusade that leads to crackdowns on sexual outsiders $»^{30}$. Dans notre cas, "gender panic" correspondrait à une " croisade morale pour réprimer les

29 L'ouvrage collectif dirigé par Gilbert Herdt relate les usages de cette notion : Moral Panics, Sex Panics: Fear and the Fight Over Sexual Rights, New York, New York University Press, 2009. Lire en particulier l'article de Janice M. Irvine : "Transient Felling, Sex panics and the politics of Emotion", p. 234-276.

30 Cité par John C. Berg, Teamsters and Turtles? U.S. Progressive Political Movements in the 21st Century, Boston, Rowman \& Littlefield Publishers, 2002, p. 135. 
dissidents du (et au) genre ». On pense aussi au "genre hors-la-loi » (gender outlaw $\left.{ }^{31}\right)$ défini par Kate Bornstein.

Le sujet travesti préexiste à la télévision contrairement aux sujets transsexuels et transgenres médiatisés depuis les années 1950. Rien d'étonnant donc à ce que ce dernier soit par conséquent le modèle "spontané " et probablement choisi par défaut, de l'imaginaire social comme médiaculturel. La communication ne peut composer qu'avec des variables genrées bien définies, héritées, instituées, instituantes. La télévision fait du genre et le reproduit constamment. Nous sommes bien en présence d'une " technologie du genre " au sens de Teresa de Lauretis ${ }^{32}$.

Le corpus nous mène à envisager un mode pédagogique pour désigner le processus que révèlent les tentatives répétées depuis des décennies par les personnes trans pour changer leur image dans la société via l'exposition et la tribune médiatique. Nous constatons que quatre décennies de médiatisation croissante n'ont pas suffi à l'inscription véritable des trans dans la culture commune, et ce, quels que soient les efforts des personnes pour défendre leur cause. L'effet symbolique de chaque prestation d'une personne trans ne se réduisant toujours qu'à rejouer la dramatique du changement de sexe comme éternelle première fois ${ }^{33}$. L'inventaire de tels déficits (en représentation, en médiation) donne la mesure du phénomène : déficits d'ancêtres, de mémoires, d'histoires, de théories, de sources, de récits non-binarisants et non-hétérosexualisants. L'inscription télévisuelle sur le mode pédagogique correspond ici en tout point au modèle cisgenre et hétérosexuel : des femmes et des hommes issus de la transidentité revendiquent leur «droit » à participer, reproduire le modèle hétérosexuel, l'ordre des genres, qu'ils assurent ne pas vouloir contester ni troubler. On prend ainsi peu à peu la mesure d'une transidentité co-construite, institutionnalisée par exemple entre journalistes et trans, chacun "donnant des gages " à l'autre. On ne peut plus cantonner cette co-construction aux seuls rapports entre trans et experts psychiatres.

Les identités transsexes et transgenres ont vu leur représentation évoluer au cours de la dernière décennie. Cette évolution a été le fait de personnes en grande majorité militantes ou engagées, de journalistes ou documentaristes plus curieux et peut-être plus engagés. À défaut de maîtriser « le dossier trans ", tous tentent d'en saisir les enjeux, et l'intérêt de nouvelles questions existentielles : qui suis-je au-delà du genre assigné et reproduit ? La culture inhérente à mon genre est-elle effet de domination, de soumission, ou rien de

31 Kate Bornstein, Gender Outlaw: On Men, Women and the Rest of Us, New York, Routledge, 1994 ( $1^{\text {re }}$ éd.).

32 Teresa de Lauretis, Théorie Queer et culture populaire : de Foucault à Cronenberg, traduit de l'anglais par Marie-Hélène Bourcier, Paris, La Dispute, 2007.

33 Karine Espineira, op. cit., 2008. 
tout cela? Ma place détermine-t-elle mon rôle? Mon rôle détermine-t-il mon rang dans le monde?

Placé du côté du genre dans les discours des experts comme dans les témoignages de vie des personnes trans, le sujet est le plus souvent appréhendé par sa sexualité malgré tout. Nous parlons d'une sexualité considérée comme objet d'une identité sexuelle instinctive, incontrôlée et non civilisée dans les discours moralistes religieux et parfois " psychiatriques ". N'est-ce pas contre cette inscription comme " obscur objet " que des transsexuels se défendent si souvent en télévision ? Notre corpus montre aussi que la parole des travestis des faits divers des années 1970 est souvent plus politique et plus critique que celle des transsexuels devenus l'enjeu d'une question de société dans les années 1980 et sommés de rentrer dans le rang. Effet secondaire de l'inscription de la cause trans dans le mouvement d'égalité des droits, une parole contestataire au sein même des "politiques trans " (comme définies par Marie-Hélène Bourcier en 2005) ${ }^{34}$, se dessine et s'affirme.

L'incursion vers d'autres formes d'expression possibles de l'égalité des droits, à travers une approche originale telle que la téléréalité, semble pertinente. Elle montre que la téléréalité participe à familiariser le sujet trans auprès du public. De nouvelles analyses sont désormais possibles avec la dernière vague de documentaires : Screamin Queens - The Riot at Compton's Cafeteria (VictorSilverman et Susan Stryker, 2005), L'Ordre desmots (Cynthia et MélissaArra, 2007), Nous nirons pus au bois (Josée Dayan, 2007), Diagnosing Difference (Annalise Ophelian, 2009), Mon sexe n'est pas mon genre (Valérie Mitteaux, 2011), Mes questions sur les trans (Serge Moati \& Charlotte Lessana, 2011), Identités remarquables (Emmanuelle Vilain \& Nathalie Lépinay, 2011), Bambi (Sébastien Lifshitz, 2013), pour la plupart hors corpus en raison de leur date de production/diffusion ou de leur non-télédiffusion. Notons encore, que si ces documents mettent encore en scène la confession intime ou le récit biographique pour certains d'entre eux, ils ne font plus l'impasse sur la situation politique, sociale, culturelle ou philosophique des personnes trans. Une évolution de la construction médiatique des personnes trans semble bien être amorcée.

\section{Karine Espineira \\ Chercheure associée au LIRCES, EA 3159 \\ Université de Nice Sophia Antipolis \\ karine.espineira@me.com}

34 Karine Espineira, "La médiatisation des politiques transgenres : Du statut de contre-public à l'inégalité de la représentation ", Revue Française des Sciences de l'Information et de la Communication, $\mathrm{n}^{\circ}$ 4, 2014, [en ligne] : http://rfsic.revues.org/695 [consulté le 17/09/2014] et «La sexualité des sujets transgenres et transsexuels dans les médias. Processus croisés de sursexualisation et de désexualisation des figures médiatiques trans ", Hermès, n 69 "Sexualités ", Paris, CNRS Éditions, 2014, p. 105-109. 


\section{Résumé}

Notre étude des représentations médiatiques des transidentités est centrée sur la télévision, en raison de la richesse de ses différentes formes de création et de diffusion (actualités, reportages, documentaires, débats, divertissements, fictions et cinéma). Elle s'appuie sur cinq années d'observation participative du terrain transidentitaire français (associations et collectifs transidentitaires, personnes transgenres et transsexes) et sur un corpus de plus de huit cents documents audiovisuels, constitué sur les bases archives de l'Institut National de l'Audiovisuel. Nous nous intéressons aux modélisations sociales et culturelles, causes et conséquences du processus de médiatisation. L'analyse des glissements successifs du lexique médiatique du fait-divers au fait de société, jusqu'à la notion de l'égalité des droits permet de décrire et d'étudier les liens qu'entretiennent des représentations sociales et des représentations médiatiques consubstantielles. Les logiques de production, les structures de représentation des périodes déterminées éclairent sur les dynamiques sociales en action et en interaction.

\section{Mots-clés}

Média, télévision, lexique médiatique, modèle de représentation, genre, transidentité.

\section{Abstract}

Our study of the media representations of trans-identities focuses on television, because of the wealth of its various forms of creation and broadcasting: news, reports, documentaries, debates, entertainments, fictions and cinema. It leans on five years of participant observation of the French field (transgender and transsexuals associations) and on a corpus of more than eight hundred audiovisual documents, constituted on the archival bases of the French National Audio-visual Institute. We are interested in the social and cultural modelling, causes and consequences of the process of mediatisation. The analysis of the successive shifts of the media lexicon, from the news stories to the social phenomenon and lastly the notion of equal rights, allows us to describe and to study the links which maintain social representations and consubstantial media representations. The logics of production, the structures of representation of determined periods enlighten on the social dynamics in action and interaction.

\section{Keywords}

Gender, media, television, media lexicon, transgender, models of representation. 